\title{
SISTEMA ANTICOLISÃO APLICADO A MANIPULADORES ROBÓTICOS BASEADO EM CAMPOS POTENCIAIS ARTIFICIAIS
}

\author{
Ubiratan Junior* Caio Cristiano Barros Viturino* \\ André Gustavo Scolari Conceição* Leizer Schnitman ** \\ * Departamento de Engenharia Elétrica, Universidade Federal da \\ Bahia,BA, (e-mail: eng.ele.ubiratan@gmail.com). \\ ** Departamento de Engenharia Química, Universidade Federal da \\ Bahia,BA, (e-mail: leizer@ufba.br)
}

\begin{abstract}
The development of anti-collision robotic systems has undergone several advances in algorithms of route planning. These systems, widely used in autonomous activities, generate free routes of collisions with objects in the workspace of the robot. In this context, the Artificial Potential Fields technique has been the focus of improvements in recent years due to its simplicity of application and efficiency in real-time systems, due to the fact that it does not require a global mapping of the robot's workspace. In spite of its efficiency, this technique is susceptible to local minima problems of different natures, such as: Goals Non-Reachable with Obstacles Nearby and Reacharound Local Minimum Problem. To solve these problems, an improvement called Adaptive Artificial Potential Fields is used in conjunction with the Subgoal Selection, Goal Configuration Sampling and Convex Hull techniques. The Robot Operating System (ROS) framework and a collaborative robot manipulator UR5 validate the proposed method.

Resumo: O desenvolvimento de sistemas robóticos anticolisão tem passado por diversos avanços em algoritmos de planejamento de rotas. Estes sistemas, amplamente utilizados em atividades autônomas, geram rotas livres de colisões com objetos na área de trabalho do robô. Neste contexto, a técnica dos Campos Potenciais Artificiais tem sido foco de melhorias nos últimos anos devido a sua simplicidade de aplicação e eficiência em sistemas de tempo real, pelo fato de não necessitar de um mapeamento global da área de trabalho do robô. Apesar da sua eficiência, esta técnica é suscetível a problemas de mínimos locais de naturezas diferentes, como: Goals NonReachable with Obstacles Nearby e Reacharound Local Minimum Problem. Para solucionar estes problemas, uma melhoria chamada de Campos Potenciais Artificiais Adaptativos é utilizada em conjunto com as técnicas de Subgoal Selection, Goal Configuration Sampling e Convex Hull. Resultados experimentais com o framework Robot Operating System (ROS) e um Manipulador Robótico colaborativo UR5 validam a abordagem proposta.
\end{abstract}

Keywords: Robot manipulators, Adaptive Artificial Potential Field, Path Planning.

Palavras-chaves: Robôs manipuladores, Campos Potenciais Artificiais Adaptativos, Planejamento de rotas.

\section{INTRODUÇÃO}

Desenvolvimentos tecnológicos permitem que novas gerações de robôs se tornem cada vez mais autônomas. Neste aspecto, os robôs podem ter habilidades de percepção, localização, controle e decisão própria (Leite et al., 2015). Um dos objetivos de sistemas autônomos robóticos é realizar um controle seguro de movimentação, de tal modo que não haja colisão com obstáculos, como pessoas, máquinas e objetos (Thomas et al., 2011). Tal objetivo exige tratamentos de problemas diversos como localização, controle de movimento e planejamento de trajetória e rotas.

Um exemplo de algoritmo de planejamento de rotas para aplicação, em tempo real, de um sistema anticolisão em robôs manipuladores e móveis, conhecido como Campos
Potenciais Artificiais (CPA), foi primeiramente proposto por (Khatib, 1986). Com a utilização dos CPAs, em robôs manipuladores, os elos são vistos como partículas carregadas que sofrem intervenção de campos potenciais repulsivos gerados por obstáculos e de um campo atrativo gerado pela posição final ou objetivo. Apesar da sua eficiência, os CPAs clássicos possuem algumas restrições ou mínimos locais, tais como:

- Impossibilidade de alcançar o objetivo quando o mesmo está dentro da área de influência do obstáculo, problema conhecido como Goals Non-Reachable with Obstacles Nearby (GNRON) (Ge and Cui, 2000). Este problema ocorre em robôs móveis e manipuladores. 
- Não convergência da rota nas configurações de posicionamento em que existam obstáculos próximos aos elos. As forças repulsivas do obstáculo impedem que o efetuador final alcance o objetivo, problema conhecido como Reacharound Local Minimum Problem (RLMP) (Byrne et al., 2013). Este problema ocorre exclusivamente em manipuladores robóticos.

A ascensão de pesquisas na área de planejamento de rotas que utilizam o método dos CPAs tem sido notável (Adorno, 2008). As áreas de aplicações englobam robôs manipuladores, robôs móveis, carros autônomos, veículos aéreos não tripulados (VANT), veículos autônomos subaquáticos entre outros. Alguns trabalhos nesta área são comentados a seguir:

Ge and Cui (2000) elaboraram uma técnica, nomeada de Campos Potenciais Artificiais Modificados (CPAM) para solucionar problemas de GNRON em robôs móveis. Luo et al. (2012) expandiu essa técnica para um espaço tridimensional. Esta técnica, porém, aumenta as chances de estagnação em mínimos locais.

Para solucionar este problema dos CPAMs, Zhang et al. (2017) desenvolveram chamado de Campos Potenciais Artificiais Adaptativos. Nesse método, são utilizados conceitos dos CPAs clássicos, quando se está distante do objetivo, e dos CPAMs, quando se está próximo ao objetivo. Assim, a rota percorrida não enfrenta problemas relacionados a mínimos locais causados pelos campos potenciais repulsivos e, ao mesmo tempo, é capaz de solucionar o problema de GNRON.

Kim et al. (2016) integraram os CPAs clássicos à abordagem dos cones de colisão (Collision Cone Approach), para que seja possível prever uma possível colisão entre robôs móveis e obstáculos.

Li et al. (2015) apresentaram um método de planejamento de rotas para robôs móveis em ambientes conhecidos, parcialmente conhecidos ou totalmente desconhecidos. Os CPAs foram integrados ao método Simultaneous Forward Search Method (SIFORS) para encontrar uma rota válida e curta até o objetivo.

Os CPAs possuem alguns problemas de mínimos locais característicos de robôs móveis e manipuladores. Robôs móveis têm maior liberdade de movimento, enquanto que, em uma cadeia de corpos rígidos de um manipulador, a movimentação de cada junta muda a posição da junta anterior para alcançar um objetivo determinado pelo algoritmo de planejamento de rota. Ao tentar alcançar uma posição final, um manipulador robótico pode estagnar em um mínimo local devido às forças de repulsão agindo nos elos, problema conhecido como RLMP.

Byrne et al. (2013) utilizaram os métodos conhecidos como Goal Configuration Sampling, Subgoal-Selection, fundamentados no método Sampling-based e o algoritmo Expanded Convex Hull, para evitar os mínimos locais RLMP causados pelos CPAs.

Akbaripour and Masehian (2017) desenvolveram um método que integra algoritmos Probabilistic Roadmap Method (PRM) e Lazy-PRM. Esse método foi nomeado de SemiLazy Probabilistic Roadmap Method (SLPRM) e é fundamentado em algoritmos Sampling-based. O método foi desenvolvido para aplicação em robôs manipuladores. Os resultados mostraram que a eficiência computacional do algoritmo $S L P R M$ é maior se comparado aos algoritmos PRM e Lazy-PRM.

Este trabalho tem por objetivo mostrar os resultados da aplicação de técnicas para solução dos problemas de GNRON e RLMP em manipuladores robóticos. Ele é divido da seguinte forma: A seção 1 introduz o tema de algoritmos de planejamentos de rotas com desvio de obstáculos e faz uma revisão bibliográfica de trabalhos mais recentes na área. A seção 2 exibe a teoria dos CPAs clássicos. A seção 3 trata do CPAAs como uma solução para o problema de GNRON . A seção 4 mostra uma solução para o problema de RLMP. A seção 5 evidencia os resultados da aplicação da técnica dos CPAMs aplicadas ao manipulador UR5 como solução para os problemas de RLMP e GNRON através de dois experimentos, com e sem utilização das técnicas propostas. A seção 6 conclui o artigo e discute sugestões de trabalhos futuros.

\section{CAMPOS POTENCIAIS ARTIFICIAIS CLÁSSICOS}

Os campos atrativos e repulsivos se assemelham com o conceito de campos eletrostáticos. A força que age no ponto de controle do robô é equivalente ao gradiente negativo de $U$ e é dado por:

$$
F(q)=-\nabla U(q)=-\nabla U_{a t t}(q)-\nabla U_{r e p}(q)
$$

Um dos requisitos que deve ser considerado pelo campo potencial atrativo é aumentar com a distância entre $q_{\text {final }}$ e $q_{\text {inicial }}$. Uma das alternativas para a solução do problema é a utilização de um campo que cresce quadraticamente com a distância de $q_{\text {final }}$. Sendo $\rho_{f}$ a distância Euclidiana entre $q_{\text {final }}$ e $q_{\text {inicial }}$, denotada por:

$$
\rho_{f}(q)=\left\|q-q_{\text {final }}\right\|
$$

É possível definir o campo atrativo quadrático como $\nabla U_{a t t}(q)$ tal que

$$
\nabla U_{a t t}(q)=\nabla \frac{1}{2} \varsigma \rho_{f}^{2}(q)=\varsigma(q-q \text { final })
$$

onde $\varsigma$ é um parâmetro usado para fator de escala do campo atrativo.

Nos CPAs clássicos, o campo repulsivo é anulado após certa distância de influência $\rho_{0}$ do obstáculo e o eleva quando próximo a ele, isto é,

$$
U_{r}(q)=\left\{\begin{array}{lll}
\frac{1}{2} \eta_{j}\left(\frac{1}{\rho}-\frac{1}{\rho_{0}}\right)^{2}, & \text { se } & \rho \leq \rho_{0} \\
0, & \text { se } & \rho>\rho_{0}
\end{array}\right.
$$

onde a distância de influência do obstáculo é representada por $\rho_{0}$, a menor distância de $q$ para os limites de um obstáculo no espaço de configurações é definida por $\rho$ e $\eta$ é um coeficiente de ganho que determina a influência do campo repulsivo. A expressão para força repulsiva é:

$$
F_{r e p}(q)=\left\{\begin{array}{lll}
\eta_{j}\left(\frac{1}{\rho}-\frac{1}{\rho_{0}}\right) \frac{1}{\rho^{2}} \nabla \rho, & \text { se } & \rho \leq \rho_{0} \\
0, & \text { se } & \rho>\rho_{0}
\end{array}\right.
$$

onde $\rho$ representa a relação da menor distância entre pontos de controle no robô e obstáculos e $\nabla \rho$ indica o gradiente $\nabla \rho(x)$ avaliado em $x=C_{j}(q)$. Se um ponto $b$ nas limitações do obstáculo na área de trabalho estiver 
próximo ao campo repulsivo de um ponto de controle no robô, então $\nabla \rho=\left\|c_{j}(q)-b\right\|$ e seu gradiente é representado por:

$$
\left.\nabla \rho(x)\right|_{x}=\frac{a_{j}(q)-b}{\left\|a_{j}(q)-b\right\|}
$$

As forças que agem nos pontos de controle do robô são somadas e aplicadas às juntas, através da transposta do Jacobiano, para obter o torque necessário para movimentação das juntas. A força total é definida como:

$$
\begin{gathered}
F(q)=\sum_{i} J_{i}^{T}(q) F_{a t t, i}(q)+\sum_{i} J_{i}^{T}(q) F_{r e p, i}(q) \\
\text { 3. CAMPOS POTENCIAIS ARTIFICIAIS } \\
\text { ADAPTATIVOS }
\end{gathered}
$$

Para eliminar problemas de GNRON presentes nos CPAs clássicos, Zhang et al. (2017) desenvolveram os Campos Potenciais Artificiais Adaptativos (CPAA). O campo repulsivo dos CPAAs é representado por

$$
U_{r e p}(q)=\left\{\begin{array}{lll}
\frac{1}{2} \eta_{j}\left(\frac{1}{\rho}-\frac{1}{\rho_{0}}\right)^{2} \frac{\rho_{g}^{n}}{1+\rho_{g}^{n}}, & \text { se } & \rho \leq \rho_{0} \\
0, & \text { se } & \rho>\rho_{0}
\end{array}\right.
$$

onde $n>0$.

Na Equação 8, quando $n=1$ e o robô está longe do objetivo, isto é, $\rho_{g}^{n} \gg 1$, então $\rho_{g}^{n} /\left(1+\rho_{g}^{n}\right) \approx 1$ e o campo repulsivo dos CPAAs são equivalentes aos campos repulsivos dos CPAs, evitando o caminho aumente à proporção da distância ao objetivo. Quando o robô está próximo ao objetivo, ou seja, $\rho_{g}^{n} \ll 1$, a expressão $\rho_{g}^{n} /\left(1+\rho_{g}^{n}\right)$ é equivalente à aproximadamente $\rho_{g}$. Nos CPAAs, a força repulsiva é fragmentada em outras duas componentes, as quais atraem o robô até o objetivo e repelem o robô do obstáculo, mesmo que ele esteja posicionado dentro da área de influência $\rho_{0}$, isto é,

$$
F_{r e p}(q)= \begin{cases}F_{r e p 1} n_{O R}+F_{r e p 2} n_{R G}, & \text { se } \rho \leq \rho_{0} \\ 0, & \text { se } \rho>\rho_{0}\end{cases}
$$

onde o vetor unitário $n_{O R}=\nabla \rho\left(q, q_{o b s}\right)$ indica a direção do obstáculo ao ponto de controle do robô e $n_{R G}=$ $-\nabla \rho\left(q, q_{\text {goal }}\right)$ indica a direção do robô ao objetivo. A componente $F_{\text {rep } 1}$, representada por:

$$
F_{\text {rep } 1}=\eta_{j}\left(\frac{1}{\rho}-\frac{1}{\rho_{0}}\right) \frac{\rho_{g}^{n}}{\rho^{2}\left(1+\rho_{g}^{n}\right)}
$$

repele o robô do obstáculo e a componente $F_{\text {rep } 2}$, representada por:

$$
F_{\text {rep } 2}=\frac{n}{2} \eta_{j}\left(\frac{1}{\rho}-\frac{1}{\rho_{0}}\right)^{2} \frac{\rho_{g}^{n-1}}{\left(1+\rho_{g}^{n}\right)^{2}}
$$

atrai o robô até o objetivo.

A Figura 1 mostra as componentes das forças atrativas e repulsivas dos CPAAs. A força total gerada a partir dos CPAAs guia o efetuador final do robô até o objetivo, representado pela circunferência verde.

Para que somente o efetuador final, e não os demais elos, consiga alcançar uma posição dentro do campo de influência do obstáculo, os CPAAs foram aplicados somente ao terceiro elo, enquanto que, o elo um e dois permanecem utilizando os CPAs. Isso garante que os elos um e dois não entrem na área de influência dos obstáculos.

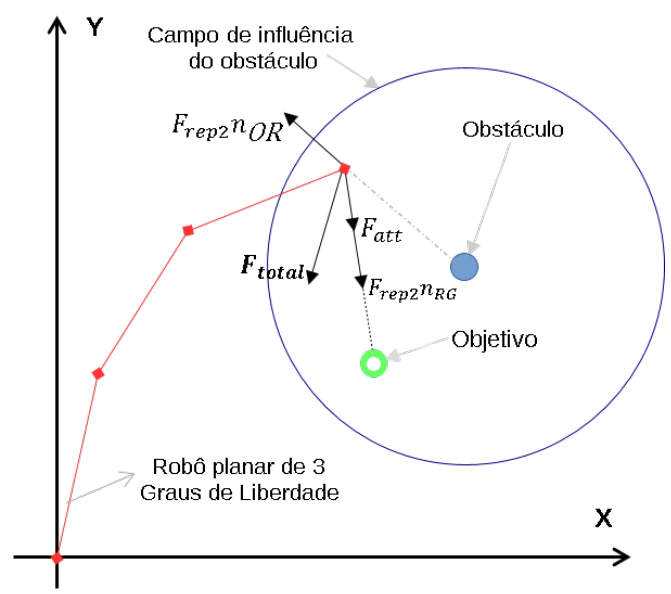

Fig. 1. Componentes das forças atrativas e repulsivas dos CPAA.

\section{REACHAROUND LOCAL MINIMUM PROBLEM}

Byrne et al. (2013) propuseram o uso do algoritmo Goal Configuration Sampling e Subgoal Selection com a finalidade de elaborar rotas válidas alternativas para que o efetuador final alcance o objetivo sem que existam problemas de RLMP.

\subsection{Goal Configuration Sampling}

O algoritmo Goal Configuration Sampling permite calcular o conjunto de configurações do robô, em que o efetuador alcança a posição final, sem que seus elos se aproximem dos campos repulsivos dos obstáculos. Usando a lei dos cossenos é possível calcular os ângulos das juntas pertencentes a esse conjunto de configurações, isto é,

$$
\theta_{i}= \pm \cos ^{-1}\left(\frac{L_{i}^{2}+J_{i} G^{2}-\left(L_{i+1}+\ldots+L_{N}\right)^{2}}{2 L_{i} J_{i} G}\right)
$$

onde $\theta_{i}$ é o conjunto de ângulos para a junta $J_{i}$ que permite ao manipulador alcançar a posição final, $J_{i} G$ representa a distância de $J_{i}$ para a posição final e $L_{i}$ representa o comprimento do elo $i$.

A Figura 2 evidencia o Goal Configuration Sampling do manipulador robótico utilizado em relação ao objetivo definido. Os ângulos obtidos consideram as limitações das juntas e os obstáculos no caminho. Desta forma, é possível obter um conjunto de configurações válidas em que o efetuador final alcança o objetivo.

Após a obtenção destas configurações possíveis, são calculadas as diferenças entre os ângulos das posições finais, presentes no Goal Configuration Sampling, e a posição inicial. A posição final é escolhida com base na menor variação encontrada, ou seja, a posição em que a rota percorrida, no espaço de juntas, será menor.

\subsection{Subgoal Selection}

É possível observar, na Fig. 3, que utilizando somente os CPAAs e o algoritmo Goal Configuration Sampling, não é possível alcançar o objetivo quando existe um ou mais obstáculos no caminho. A posição do mínimo local mostra que o obstáculo exerce forças repulsivas nos elos, 


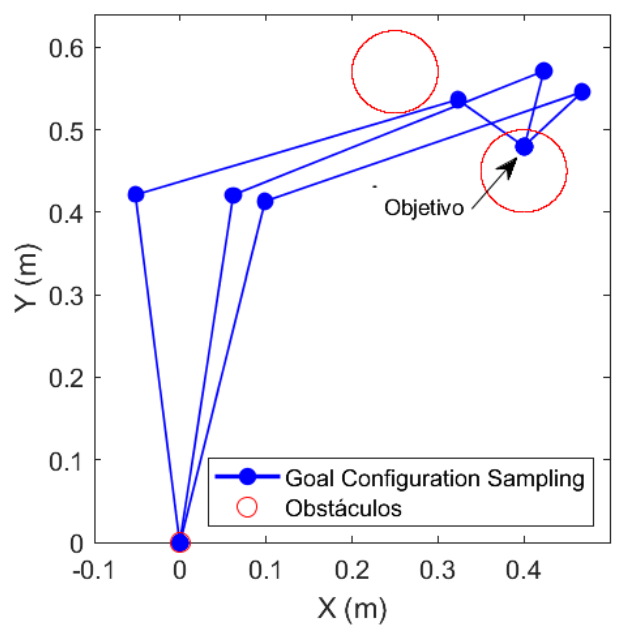

Fig. 2. Goal Configuration Sampling gerado através da lei dos cossenos.

interrompendo seu movimento, porém, com o uso dos algoritmo Subgoal Selection é possível uma rota válida alternativa para que o manipulador alcance o objetivo, como mostra a Fig. 4.

A rota criada pelo algoritmo Subgoal Selection contempla os pontos intermediários criados pelo algoritmo Convex Hull entre o objetivo, posição inicial do efetuador e os obstáculos no caminho.

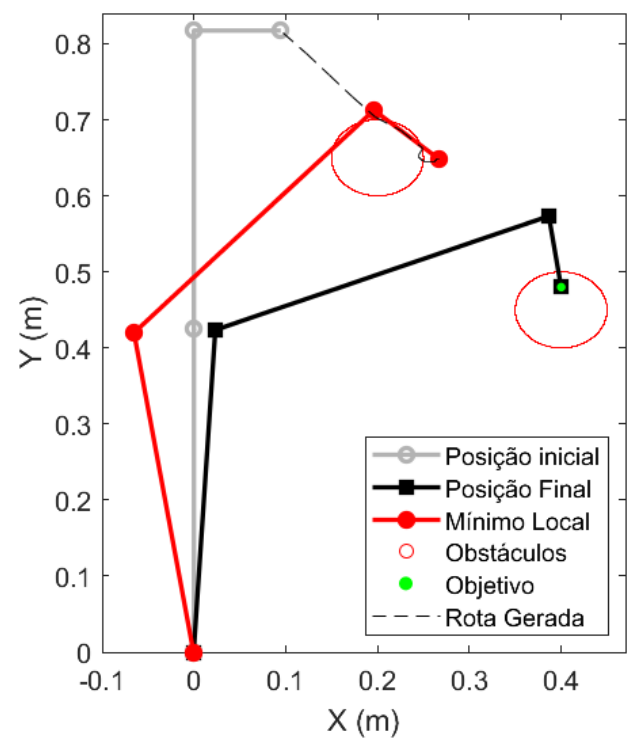

Fig. 3. Planejamento de rotas utilizando somente os CPAAs. O problema de RLMP não permite que o manipulador alcance o objetivo.

O Algoritmo 1 mostra o funcionamento do Goal Configuration Sampling, em conjunto com Subgoal Selection e os CPAAs. Os algoritmos Goal Configuration Sampling e Subgoal Selection são utilizados somente quando as rotas geradas pelos CPAAs não são válidas, ou seja, quando obstáculos estão posicionados no caminho até o objetivo. Em caso de existência de uma rota válida, somente os CPAAs são utilizados.

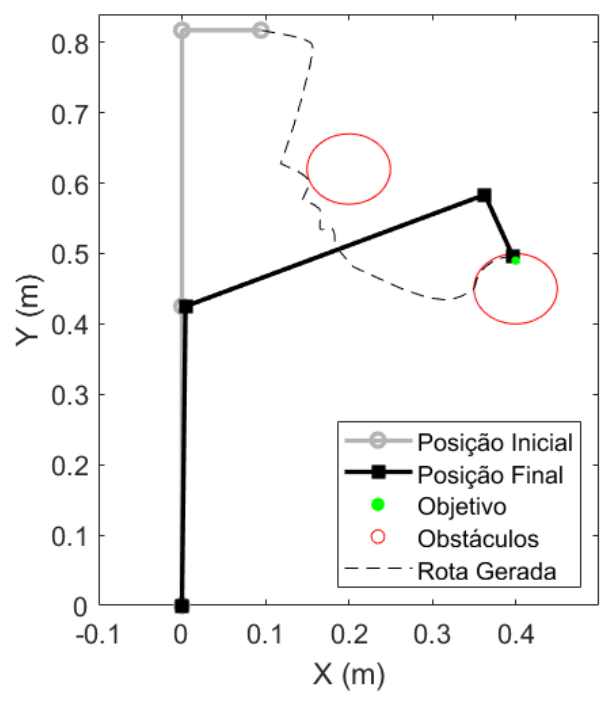

Fig. 4. Planejamento de rotas utilizando Goal Configuration Sampling, Subgoal Selection e CPAA

\section{RESUlTAdOS E DISCUSSÕES}

Diversos testes práticos foram realizados no manipulador robótico UR5 da Universal Robots utilizando os algoritmos propostos neste trabalho, Fig. 8, e os CPAs clássicos, Fig. 5, para fins de comparação. Os resultados mostraram que, utilizando somente os CPAs clássicos, não é possível alcançar o objetivo, quando há obstáculos entre a posição inicial e final do manipulador. Em ambos os experimentos, a posição inicial $q_{i}=[0,-1.57,0,0,0,-1.57]^{T}$, em radianos, as coordenadas dos obstáculos $P_{o b s_{1}}=[0.2,0.11,0.72]^{T}$ e $P_{o b s_{2}}=[0.4,0.11,0.55]^{T}$, em metros, e do objetivo $P_{o b j}=[0.4,0.11,0.59]^{T}$, em metros, foram mantidas as mesmas. A configuração final do manipulador para se alcançar o objetivo é $q_{f}=[0,-1.56,1.14,0.05,0,-2.77]^{T}$, em radianos.

A Figura 6 evidencia o deslocamento das juntas dois, três, quatro e seis do robô UR5 utilizando os Campos Potenciais Artificiais clássicos. A Figura 7 mostra o gradiente negativo dos CPAs clássicos quando o mesmo não alcança o objetivo devido ao problema de RLMP. É possível notar que as forças atrativas e repulsivas são aproximadamente iguais em módulo e, consequentemente, se anulam. No tempo equivalente a 5 segundos, é possível notar que o manipulador já se aproxima da posição de mínimo local, representado na Figura 5

\begin{tabular}{l}
\hline Algoritmo 1 \\
\hline Entrada $\theta_{i}, x_{\text {final }}, y_{\text {final }}, \zeta, \alpha, \rho_{0}, \eta$ \\
Validar a rota gerada pelos CPAAs \\
Se Não existir uma rota válida Então \\
Utilizar o algoritmo Goal Configuration Sampling \\
Utilizar o algoritmo Subgoal Selection \\
Aplicar os CPAAs para guiar o efetuador final até o \\
objetivo \\
Se não \\
Utilizar somente os CPAAs para guiar o efetuador \\
final até o objetivo \\
Fim Se \\
\hline
\end{tabular}



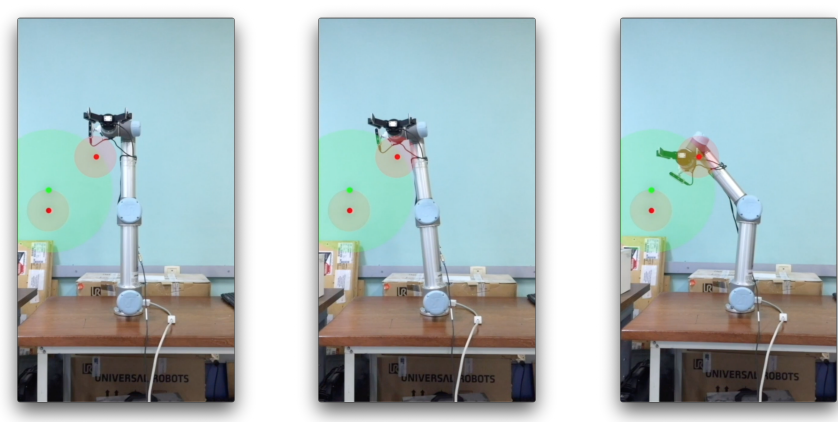

Fig. 5. Aplicação dos CPAs Clássicos (ocorrência de mínimo local)

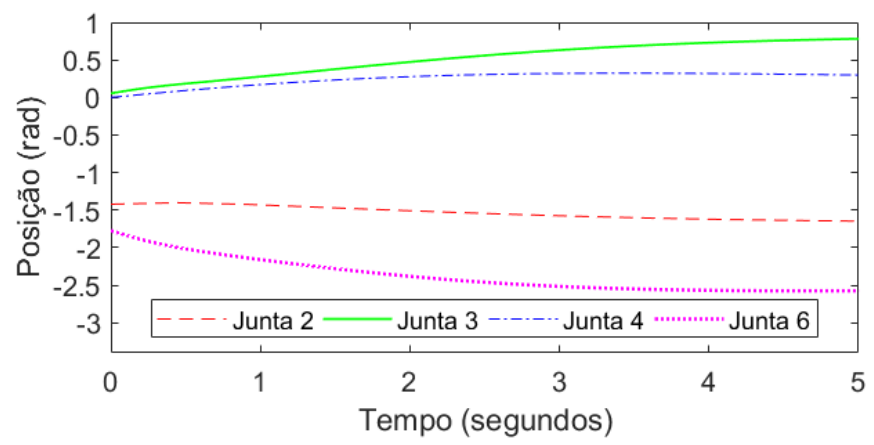

Fig. 6. Posição das juntas após a aplicação dos Campos Potenciais Artificiais clássicos
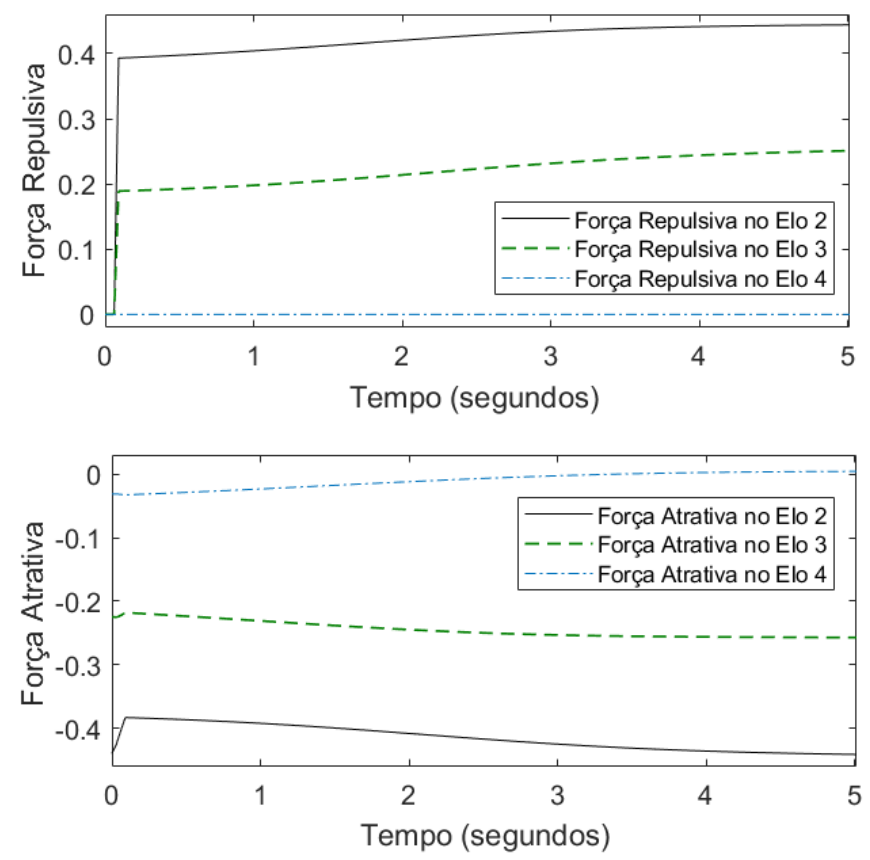

Fig. 7. Forças repulsivas e atrativas dos Campos Potenciais Artificiais clássicos

A Figura 8 mostra as posições do robô UR5 quando método proposto é utilizado. Resultados comprovam que é possível produzir movimentos rápidos, enquanto o robô é guiado até a posição final, desviando de obstáculos localmente.
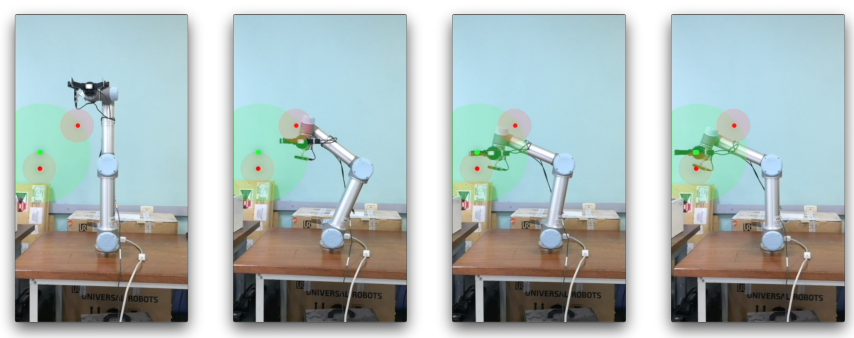

Fig. 8. Aplicação do método proposto (desvio do mínimo local)

A Figura 9 evidencia o deslocamento das juntas dois, três, quatro e seis do robô, da posição inicial até a posição final. Enquanto a Figura 10 mostra a posição cartesiana para o experimento utilizando o método proposto neste trabalho. A partir desses gráficos é possível observar que os movimentos executados são suaves tanto no espaço de juntas, quanto no espaço cartesiano. Nestes experimentos, a orientação do efetuador foi mantida constante, como pode ser visto nas Figuras 5 e 8 .

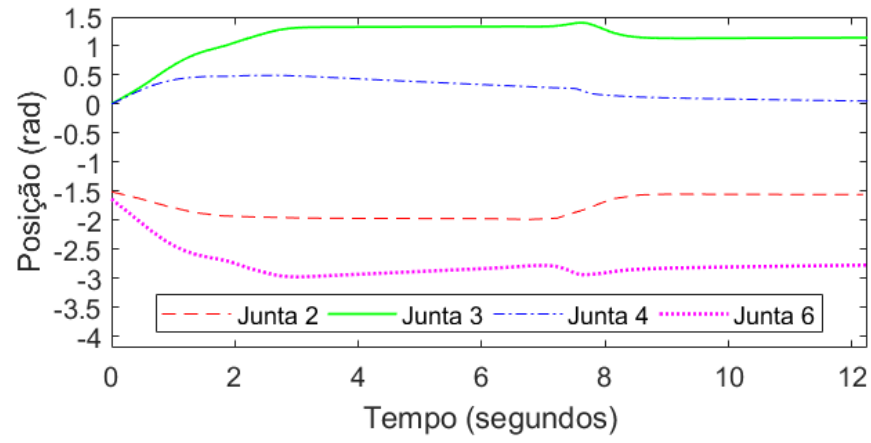

Fig. 9. Posição das juntas após a aplicação dos Campos Potenciais Artificiais Adaptativos, Goal Configuration Sampling e Subgoal Selection

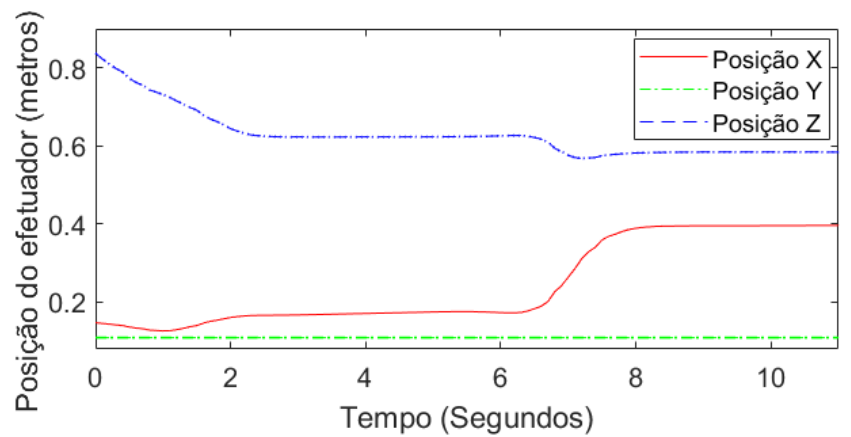

Fig. 10. Posição do efetuador final em relação à base do UR5

A Figura 11 mostra o gradiente negativo dos CPAAs sobre os elos do manipulador robótico UR5. No tempo equivalente a 7.8 segundos, o elo 4 se aproxima de um campo repulsivo e nota-se uma mudança abrupta da força repulsiva e atrativa, porém, o manipulador consegue se desviar e alcançar o objetivo devido aos algoritmos Subgoal Selection e Goal Configuration Sampling.

A Tabela 1 resume os métodos utilizados em cada experimento e os resultados alcançados. 

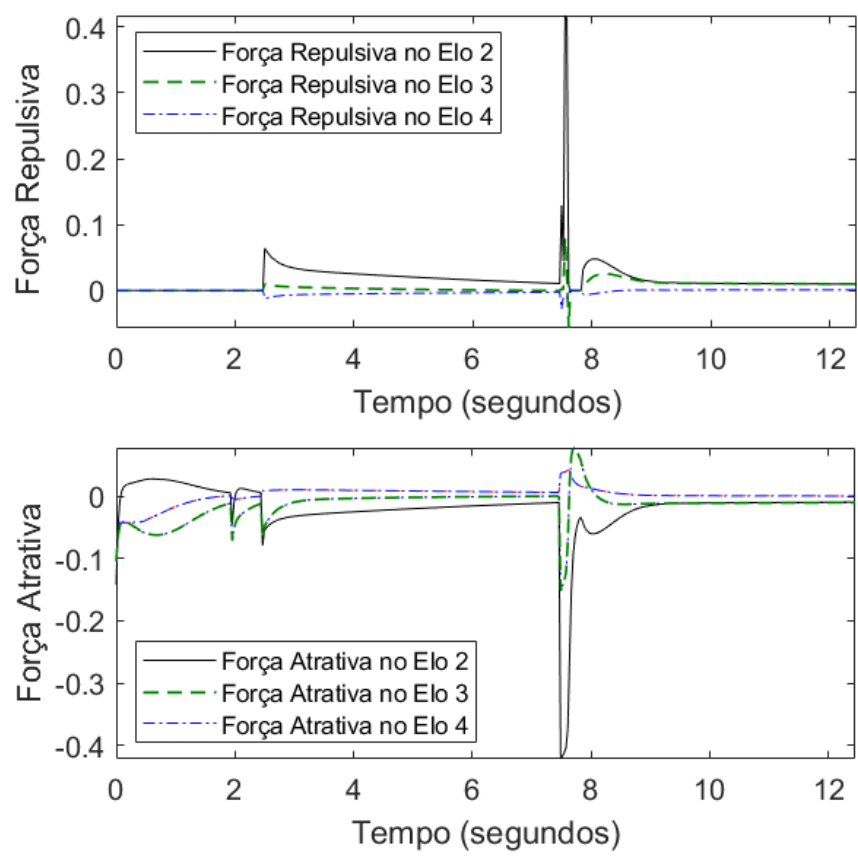

Fig. 11. Forças repulsivas e atrativas dos Campos Potenciais Artificiais utilizando o método proposto

A execução dos experimentos no UR5 pode ser vista no seguinte link: https://youtu.be/cJNlgAKH7ko.

\section{CONCLUSÕES}

Neste trabalho, foram propostas melhorias dos CPAs clássicos aplicados em manipuladores robóticos. Os mínimos locais, conhecidos como Goals Non-Reachable with Obstacles Nearby, foram evitados a partir da utilização dos Campos Potenciais Artificiais Adaptativos. Foi mostrado que os Campos Potenciais Artificiais Adaptativos não geram rotas alternativas até o objetivo, em casos onde o efetuador final não o alcança devido às forças de repulsão dos obstáculos nos elos do robô, problema conhecido como Reacharound Local Minimum Problem. Com o propósito de solucionar este mínimo local, foram integrados aos CPAAs, os algoritmos Goal Configuration Sampling e Subgoal Selection.

Apesar dos resultados obtidos, o manipulador nem sempre alcança o objetivo devido aos pesos estáticos das forças atrativas e repulsivas. É necessário que os valores dessas forças sejam atualizadas, constantemente, para que haja um equilíbrio nos campos potenciais. O método proposto também não inclui algoritmos de suavização da rota. Em trabalhos futuros, técnicas de suavização de rotas serão consideradas para suprimir oscilações geradas nas mediações dos obstáculos, devido aos cálculos de força de repulsão e atração.

Tabela 1. Métodos utilizados em testes comparativos

\begin{tabular}{ccc} 
& Figura 3 & Figura 4 \\
\hline CPA & Sim & - \\
CPAA & - & Sim \\
Goal Configuration Sampling & - & Sim \\
Subgoal Selection & - & Sim \\
Minimo global alcançado & - & Sim \\
\hline
\end{tabular}

\section{AGRADECIMENTOS}

Os autores agradecem ao apoio financeiro da FAPESB (Fundação de Amparo Pesquisa do Estado da Bahia).

\section{REFERÊNCIAS}

Adorno, B.V. (2008). Planejamento Probabilístico de Rotas no Espaço de Configuração e sua Aplicação em Robótica Móvel. Ph.D. thesis, Faculdade de Tecnologia da Universidade de Brasília.

Akbaripour, H. and Masehian, E. (2017). Semi-lazy probabilistic roadmap: a parameter-tuned, resilient and robust path planning method for manipulator robots. The International Journal of Advanced Manufacturing Technology, 89(5-8), 1401-1430.

Byrne, S., Naeem, W., and Ferguson, S. (2013). An intelligent configuration-sampling based local motion planner for robotic manipulators. In Robot Motion and Control (RoMoCo), 2013 9th Workshop on, 147-153. IEEE.

Ge, S.S. and Cui, Y.J. (2000). New potential functions for mobile robot path planning. IEEE Transactions on robotics and automation, 16(5), 615-620.

Khatib, O. (1986). Real-time obstacle avoidance for manipulators and mobile robots. The international journal of robotics research, 5(1), 90-98.

Kim, Y.H., Son, W.S., Park, J.B., and Yoon, T.S. (2016). Smooth path planning by fusion of artificial potential field method and collision cone approach. In MATEC Web of Conferences, volume 75, 05004. EDP Sciences.

Leite, A.C., Almeida-Antonio, T.B., From, P.J., Lizarralde, F., and Hsu, L. (2015). Control and obstacle collision avoidance method applied to human-robot interaction. In Advanced Robotics and its Social Impacts (ARSO), 2015 IEEE International Workshop on, 1-8. IEEE.

Li, G., Tong, S., Cong, F., Yamashita, A., and Asama, H. (2015). Improved artificial potential field-based simultaneous forward search method for robot path planning in complex environment. In System Integration (SII), 2015 IEEE/SICE International Symposium on, 760-765. IEEE.

Luo, J., Su, W., and Wang, D. (2012). The improvement of the artificial potential field robot path planning based on 3-d space.

Thomas, C., Busch, F., Kuhlenkoetter, B., and Deuse, J. (2011). Process and human safety in human-robotinteraction-a hybrid assistance system for welding applications. In International Conference on Intelligent Robotics and Applications, 112-121. Springer.

Zhang, Y., Liu, Z., and Chang, L. (2017). A new adaptive artificial potential field and rolling window method for mobile robot path planning. In Control And Decision Conference (CCDC), 2017 29th Chinese, 7144-7148. IEEE. 